\title{
VISUAL ANALYSIS OF INCONSISTENCIES IN HYDRAULIC SIMULATION DATA
}

\author{
O. Perrin ${ }^{1}$, S. Christophe ${ }^{2, *}$, F. Jacquinod ${ }^{3}$, O. Payrastre ${ }^{4}$ \\ ${ }^{1}$ Ecole Centrale de Nantes, France - octave.perrin-de-brichambaut@eleves.ec-nantes.fr \\ ${ }^{2}$ LASTIG, Univ Gustave Eiffel, ENSG, IGN, F-94160 Saint-Mande, France - sidonie.christophe@ign.fr \\ ${ }^{3}$ LASTIG, Univ Gustave Eiffel, EIVP, F-75019 Paris, France - florence.jacquinod@eivp-paris.fr \\ ${ }^{4}$ GERS-LEE, Univ Gustave Eiffel, IFSTTAR, F-44344 Bouguenais, France - olivier.payrastre@ univ-eiffel.fr
}

\section{Commission IV, WG IV/9}

KEY WORDS: Geovisualization, Simulation, Visual analysis, Flash flood, hydraulics data, Hydraulics.

\begin{abstract}
:
We present our contribution to the geovisualization and visual analysis of hydraulic simulation data, based on an interdisciplinary research work undertaken by researchers in geographic information sciences and in hydraulics. The positive feedback loop between researchers favored the proposal of visualization tools enabling visual reasoning on hydraulic simulated data so as to infer knowledge on the simulation model. We interactively explore and design 2D multi-scale styles to render hydraulic simulated data, in order to support the identification over large simulation domains of possible local inconsistencies related to input simulation data, simulation parameters or simulation workflow. Models have been implemented into QGIS and are reusable for other input data and territories.
\end{abstract}

\section{INTRODUCTION}

Given the increasing number of catastrophic inundations and the damages they inflict on populations and territories, flood mitigation policies are to be strengthened. In the European Union, a framework has been developed to enhance mitigation and adaptation strategies (EU directive 2007/60/EC) ${ }^{1}$. The efficiency of those strategies notably depends on the production of accurate knowledge about flood hazards and risks, to improve prevention and management of related crisis. Particularly, the knowledge about risks related to flash-floods of small rivers threatening urban areas has still to be improved. Providing databases of flood simulations with different discharge return periods at a national level is a way to evaluate possible impacts of flash floods. Producing such knowledge remains a challenging issue due to the large network of small rivers possibly affected, and the scarcity and poor quality of available input data. In France, one purpose of the PICS Project ${ }^{2}$ is to automatically produce flood hazard maps for small rivers on large territories, based on a Digital Terrain Model (DTM) input data. Automation of the hydraulic simulation process over large and varied territories, and heterogeneous quality of the terrain input data, require numerous and iterative analysis of simulation results in order to propose a simulation method that will provide the most accurate results at a very large scale.

Identification of changes, patterns, breaks in output data would help specify simulation limits and related ways of improvement, regarding input data, simulation parameters or simulation workflow. A visual analytics approach is required here, in order to favor visual interpretation and reasoning on data simulation. This approach needs a close collaboration between researchers in hydraulics and in GI sciences, in order to iterate regularly throughout the visualization design process so as to adapt the propositions to the users needs. Hydraulic researchers do not have a pre-established idea of the main error locations and/or

\footnotetext{
* Corresponding author

1 https://ec.europa.eu/environment/water/flood ${ }_{r} i s k /$

2 http://pics.ifsttar.fr/en
}

sources, so they have to explore their results and test various simulation parameters. On their end, researchers in GI sciences have to explore how geovisualization design techniques can be applied in this specific context, by making use of geovisualization tools and techniques, proposing methods to hydraulic researchers and taking into account their feedback. In our case, a satisfying proposal is one that is useful to analyze results from many different simulation methods over large simulation domains, and easy enough to use, although hydraulics researchers are not GI specialists. This paper presents the result of this positive feedback loop between researchers, i.e. the proposal of visualization tools enabling visual reasoning on hydraulic simulated data so as to infer knowledge on the simulation model.

Through this dynamic interdisciplinary process between hydraulic data simulation and visualization, we contribute to the study of the use of geovisualization for scientific simulation and its underlying issue: how to improve the visual understanding of complex dynamics on earth? The originality of our approach is to develop geovisualizations mixing cartographic visualization of basic geodata (especially DTMs) with scientific visualization of more abstract simulation results. We thus propose models of graphic representation specifically designed for the analysis of hydraulic simulation results by researchers producing those models.

After a review of literature about map design and geovisualization of flood risks, we present different inconsistencies, found in hydraulic simulation data produced by the PICS project, that are relevant to be visually detected and analyzed. In order to assist researchers in the production of simulation data, geovisualization researchers design specific graphic styles, allowing hydraulic specialists to visually analyze their simulation results, so as to be able to identify ways of improving their simulation (input data or simulation parameters) or to detect limitations of their simulation workflow. Hereafter, geovisualization researchers propose a set of automated multi-scale rendering styles for simulated data, that can be easily used by hydraulic researchers, in order to visually detect those inconsistencies, according to their needs and expectations. We finally discuss 
about the possibility to transfer these styles to $3 \mathrm{D}$, to enrich the detection and analysis of artifacts into simulated data.

\section{RELATED WORKS IN FLOOD RISKS GEOVISUALIZATION}

In the field of flood risks, there is a diversity of possible graphic representations and visualizations, among which users have to choose in relation to their purposes, expectations and the considered aspects of this complex spatio-temporal phenomenon: impacts, temporality and raising dynamics, hazard intensity (velocities, water heights), hazard probability, uncertainties, etc. As a classical geovisualization issue, the usability of visualizations will strongly depend on the matching between the final use, the graphic representation and rendering styles as well as the data exploration capacities.

Many scientific works propose ways of visualizing flood risks, but are mainly aimed at representing risk defined as "a threat to people and the things they value", as stated by (Kostelnick et al., 2013) in their proposition of a framework for risk visualization, trying to encompass all issues to be tackled for cartographic representation of risks. Such a definition of flood risks implies the representation of results from the processing of multiple data about risk so as to qualify its impact on a population or territory, whereas we are aiming herein at representing raw simulation data during their production phase and before they are passed on to any stakeholder. Most works are designed for practitioners and/or citizens (Lieske, 2012, Lonergan, Hedley, 2015, Yang, 2016, Jacquinod et al., 2016, Seipel, Lim, 2017, Massaâbi et al., 2018, Kilsedar et al., 2019, Carrillo et al., 2019). Many works explore the co-visualization of heterogeneous spatio-temporal data, thematic and topographic, for tsunami crisis management for instance (Pierkot et al., 2019) or for a better understanding of coastal dynamics (Masse, Christophe, 2015).

In addition, the users of the geovisualization outputs, i.e. the hydraulic researchers, need to visually analyze both their raw simulated results, i.e. looking for breaks, border effects, or any other unlikely results, as well as several characteristics of the considered territory (topography, vegetation, built environment), in order to spot and evaluate potential inaccuracies in their simulation models. In such a context, geovisualization issues that are usually tackled in other flood risks related scientific works need to be adapted to our specific objectives.

Graphic representation of simulation data, at any usable scale, is clearly at stake here. Graphic representation choices depend on the characteristics of the phenomenon to visualize, and on the goal of the user: this fitness for use is a classical issue for visualization design. The characteristics could be static or dynamic aspects of the phenomenon, its spatiality, its temporality, its impacts, the underlying probabilities of something happening or several kinds of uncertainties. Works focusing on representing uncertainties from simulation's results are developed for practitioners in a decision making context and citizens for awareness raising and rarely concern the direct representation of raw simulation results (Goda, Song, 2016, Lim, 2018, Klockow-McClain et al., 2020). The main difficulty regards the decision-making process which is an on-going cognitive issue for geovisualization (Padilla et al., 2018, Kübler et al., 2019). Visual variables, mainly in such context, colors, transparency, textures could be used to make something salient, and to support visual attention. Some works alternatively propose the visualization of analytics dashboards synthesizing flood risks data and socio-economic data for flood managers, thus geovisualizing processed data (Saha et al., 2018).

3D geovisualizations can allow for a better understanding of a spatial phenomenon, especially visualized on top of a 3D representation of the territory (Brasebin et al., 2016). 3D geovisualizations have already been used in flood mitigation planning with citizens, shedding light on their ability to support understanding of flood risks, because it enhances relief and water flows volume perception (Jacquinod, Bonaccorsi, 2019). Visualizing hydraulic data on top of a 3D cartographic representation of the territory based on simple GIS data (DTM, aerial images and schematic 3D buildings) has proven to be useful for hydraulic specialists and risk managers to evaluate the quality of their data and the potential damage flood could impose on this territory (Jacquinod, 2014). However, the objectives and the case studies considered here highly differ, for instance in terms of range of scales of analysis (varying here from a very large to very small scale), and of nature of information to be visualized (including internal model variables). Furthermore, as far as 3D geovisualizations are concerned, there is no consensus over which level of detail is relevant for a particular use (Bishop, Lange, 2005, Jacquinod, Bonaccorsi, 2019). Moreover 3D geovisualizations of technical data are often proposed, sometimes tested on a use case with users, but user studies necessary to generalize findings are rarely conducted (Sheppard, 2005, Kemec et al., 2010, Patel et al., 2013, Leskens et al., 2017).

Direct and interactive visualization of simulation results are also investigated, allowing stakeholders to run simulation and explore results during meetings (Leskens et al., 2014, Leskens et al., 2017), without tackling the quality of the simulation results, having rather focused on testing the usability of their user interface, which is aimed at practitioners and not hydraulic specialists.

Finally, although many works exists proposing geovisualizations of data about risks, they do not offer solutions for interactive visualizations of raw simulation data for hydraulic specialists working, in order to improve their simulation models, thus not aimed at communicating simulation results. Bringing simulation and visualization closer remains difficult for visual reasoning purposes on specific spatial configuration and phenomena.

\section{CASE STUDY AND MAIN ISSUES}

The simulation framework developed in the ANR PICS project $^{3}$ aims at providing generalized flood hazard information on small rivers over large regions, such knowledge being currently unavailable. Flood data shall be obtained for a very large number of rivers at a regional scale, which requires a high level of automation and simplification of the hydraulic simulation workflow. For this reason, hydraulic computations are run in steady state regime for different peak discharge return periods, using directly high resolution DTMs as input topographic information, and without any model calibration. To limit the extent of the computation domains, simulations are run separately on each branch of the river network, and are then combined to obtain a mono-frequency inundation scenario covering the whole

\footnotetext{
3 http://pics.ifsttar.fr/en
} 


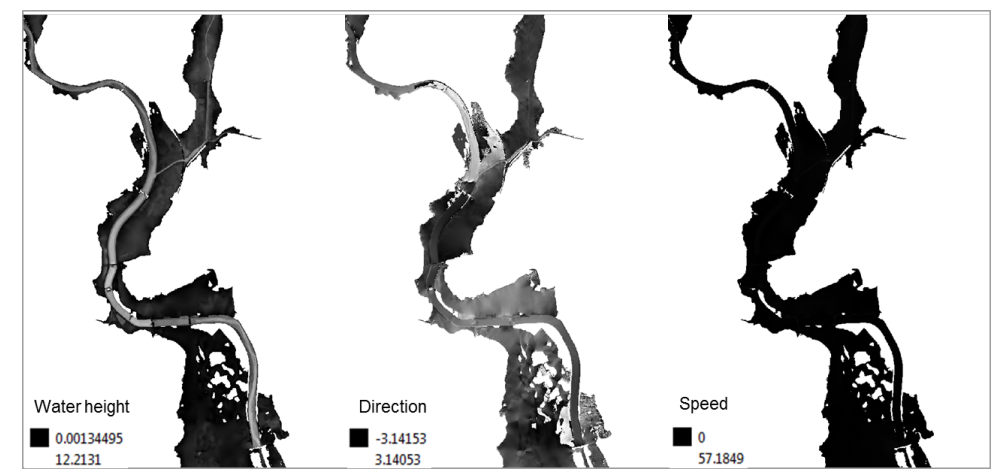

Figure 1. Visualization of raw simulated data with a grey colormap: water heights, flow directions and velocities (Alès in the Gardon watershed), with QGIS.

hydrographic network. The large simplifications and assumptions introduced in the simulation workflow generate significant errors and inconsistencies in the simulation results, which should be reduced as much as possible. Thus, the main aim of the expected visualization tools is to help hydraulics researchers in the diagnosis of their simulation results and the identification of the main error locations and sources.

The simulation results presented hereafter as an illustration of the approach were obtained in the region of Alès in the Gardon watershed, south-eastern France. This region was selected because it is frequently hit by intense flash floods affecting small rivers. It was identified as one of the main flood risk areas in France during the first application of the EU flood directive. The simulation domain considered covers a 11640x22865 m area. It includes $38 \mathrm{~km}$ of rivers among which the Gardon river and one of its small tributaries crossing the town of Alès. Hydraulic simulations are derived from an automatic application of the Floodos hydrodynamic model (Davy et al., 2017). Three successive simulations are necessary to cover the entire simulation domain; they are partly overlaying at their connection points and have to be combined to obtain the final result. The DTM used was produced in 2007 by the Conseil Départemental $\mathrm{du}$ Gard. It has a $5 \mathrm{~m}$ resolution and its estimated altimetric accuracy is $20 \mathrm{~cm}$ in non-vegetated areas, and $1 \mathrm{~m}$ in vegetated areas. However, it suffers locally from artifacts which may largely affect the simulation (such as for instance bridges not cleaned up). The simulation outputs include three raster fields (water height, flow direction, flow velocity), covering the simulation domain at a $5 \mathrm{~m}$ resolution (Fig.1).

\section{VISUAL DETECTION OF INCONSISTENCIES IN HYDRAULIC SIMULATION RESULTS}

For hydraulic simulations, researchers generally examine three types of output variables, i.e. water heights, flow directions and velocities, and are used to observe them mainly graphically with the help of a Geographic Information System (GIS), overlayed on a DTM. Without any style parameters, default visualization is often proposed to the user with levels of grey matching raster quantitative data (Fig. 1). This way of visualizing and exploring simulated data can benefit from more sophisticated graphic representation to support visual reasoning and data exploration, through scales.

As geovisualization and hydraulics researchers, we propose the following methodology for the exploratory visual analysis of hydraulic simulation results. In practice, these four following steps are not linear, this process is iterative and regular, working as a positive feedback loop.

1. Identification of inconsistencies that could be relevant for hydraulic simulation analysis, since hydraulics researchers cannot anticipate all possible flaws in their results, but may nevertheless have a preliminary idea of the possible error sources they would like to detect.

2. Experimentation of suitable visualizations by geovisualization researchers, to support visual attention on and exploration of these inconsistencies, at various scales.

3. Auto-evaluation by the hydraulics researchers based on their insights on the relevancy of the proposed visualizations for their work on simulation workflows.

4. Selection and co-design of some visualizations, then refined and automated, to be used for visual hydraulic simulation analysis.

\subsection{Characterization of inconsistencies}

Inconsistencies The term 'inconsistency' is here used to describe something that is or looks, surprising, unexpected, unexplained, possibly false, regarding the data, the model or the visualization of the data. Inconsistencies may come from different sources. Some are caused by shortcomings in the input data of the hydraulic simulation model, among which the terrain model: for instance bridges not cleaned up, or local errors due to vegetation and affecting the shape of the river bed cross section. Some are coming from the choices in terms of graphic representation of the data: unsuitable colormap classification or wrong shading of the terrain leading to mistaken visual interpretations. Issues about graphic representation may be solved by a better use of visual variables to explore data, i.e. by improving the use of color for classifications, or by adapting the parameters of terrain shading. Even if we do not focus on the solving of these particular inconsistencies, graphic representation and relief perception will be at stake hereafter.

Artifacts of interest In this paper, we focus on artifacts due to the automation and simplification of the hydraulic simulation process and to local shortcomings in the terrain model (Fig.2). They are mainly correlated with:

- Unusually high heights and speeds caused by the injection of discharge at specific points, which can only be interpreted by looking at directions. 

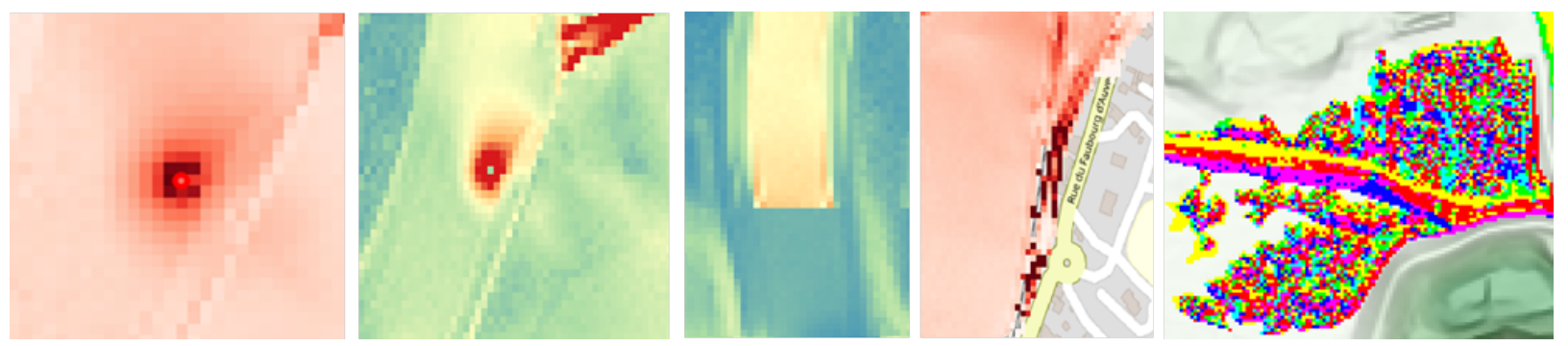

Figure 2. Examples of inconsistencies visually appearing in hydraulic simulated data. From left to right: local water level increase, sudden breaks in water levels or flow direction and velocities, apparently chaotic flow directions

- Inconsistent flow directions due for instance to the inappropriate overlaying of successive simulations at confluences: we can though observe surprising opposite directions on some areas, with no topological explanations.

- Breaks into data simulation and marginal effects often due to the terrain model.

\subsection{Issues for visual analysis of simulated data}

We focus on the visualization of the above mentioned inconsistencies: our objective is to render them more saliently to support suitable visual attention and exploration, at any visualization scales related to data resolution and size of the computation domain. We consider that the proposition should help the user: to focus on an inconsistency or a set of inconsistencies, to consider them in their context by selecting or adding other data if needed, and to zoom into the data, in order to refine the visual analysis, at a greater spatial scale, such as also accessing to hidden semantics. This implies:

- the processing of output data simulation, to create more meaningful and useful data: water heights, directions and velocities are used to be handled and visualized separately, when directions and speeds could be computed together to create a new information to render, for instance.

- the choice of relevant graphic representations for the data, i.e. selection of visual variables adapted to the characteristics of the data, the visualization scale and the other data to co-visualize: this implies to consider the notion of style.

- the evolution of the graphic representation through the scale navigation.

\subsection{A particular case study of stylization: exploring hy- draulic simulated data at a given scale}

We present here a specific case study of stylization we faced, while exploring hydraulic simulated data at a particular scale. More precisely, representing the current with the help of arrows, requires some refinements, in order to improve the interpretability of the water direction:a color wheel is presented here to support a better visual analysis of the current.

At a small scale, showing the current with arrows becomes arduous because those arrows are either too small and can't be read, or they represent too large an area over which the flow of the water may vary a lot. The first idea is to decompose the speed vector in two vectors, one aligned with the north-south axis, the other aligned with the east-west axis. Assigning a color gradient to the value of each vector resulted in a color map showing the components of the speed in both axis, which indirectly gives the direction of the current (Fig. 3.1.). This methods has some drawbacks:

- It is hard to identify the direction of the flow in low current area because the result depends on the speed of the water.

- The black is not still water but water flowing rapidly to the south-west, which is counter-intuitive.

- If the two components are outside the range of observation, the method becomes unreliable because the color is capped.
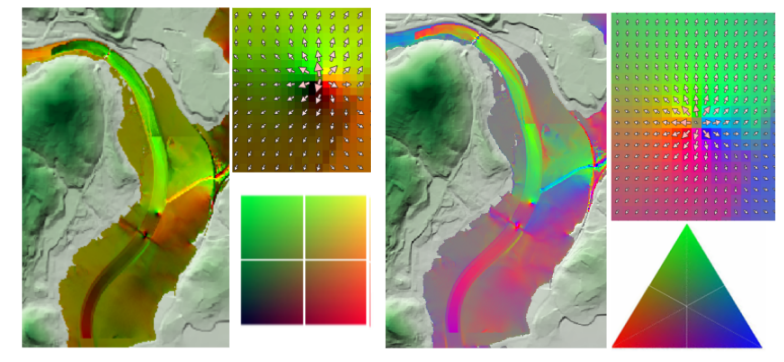

Figure 3. Stylization for current direction representation, based on a color wheel: direction is linked to hue color. (1) Left: Color gradient applied to the water speed decomposed into 2 axis (north-south and east-west), giving the direction of the current; (2) Right: Color gradient applied to the water speed decomposed into 3 axis (north, south-east, south-west).

Our proposition consists in replacing the two-axis decomposition with a three-axis decomposition, the axis used being North, South-East and South-West. Thanks to this new decomposition, fast currents are in flashy colors while still water is in gray. The imprecision of the direction for being out of the observation range is reduced (from $45^{\circ}$ to $30^{\circ}$ ) (Fig. 3.2.).

The last step is to link directly the direction of the current with the hue of the color while the saturation and the value remain maxed. This method no longer suffers from the imprecision of the direction when the current is out of the observation range. This method does not differentiate still and flowing water: the direction of the still water is shown even though it is meaningless.

The visualization finally obtained helps hydraulic researchers to focus the analysis on areas with significant flow velocities and to easily detect sudden changes in flow directions, which may appear as inconsistent. 


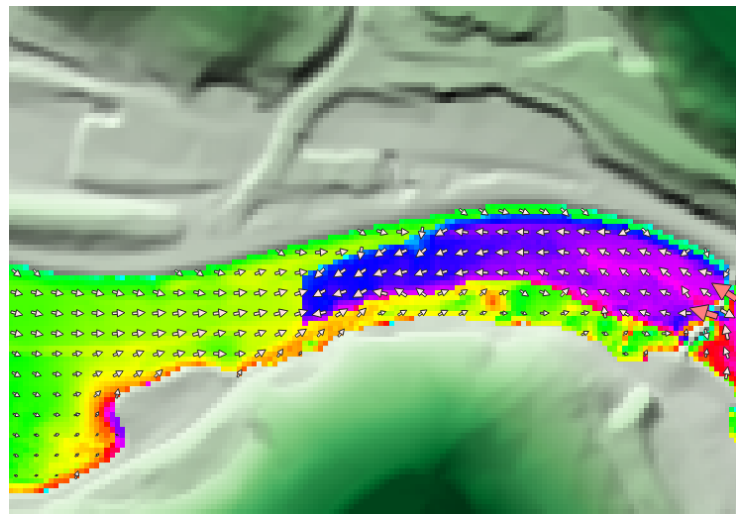

Figure 4. Actual simulation error detected by the proposed current stylization method.

This is illustrated on Fig. 4 (zoom into Fig.3), which represents here a real simulation error due to bad limit conditions management in the area where simulations are overlaying.

\section{AUTOMATED MULTI-SCALE STYLES FOR HYDRAULIC SIMULATION RESULTS}

Multi-scales styles to support the visual analysis of hydraulic simulation results, through scales, are needed: as showed in the previous example, it is important for hydraulics researchers to be able to zoom in and out the data, in order to properly analyze some inconsistencies. This section presents the design of the set of graphic multi-scale styles, and their automation in QGIS.

\subsection{A set of graphic styles through scales}

After several iterations between geovisualization and hydraulics researchers, we developed a multi-scale approach in order to highlight relevant inconsistencies and to enable data assessment, throughout scales. A multi-scale geovisualization encompassing different graphic representation styles for each relevant scale has been formalized and can be used on simulation results seamlessly at any scale, so that the identified inconsistencies could be spotted on any dataset, small or large. Those graphic styles are designed to facilitate the search for inconsistencies and their origin, which often requires many zooming in and out operations and simultaneous display of several datasets.

Figure 5 presents this multi-scale approach, based on several graphic styles, which relies on the visualization of the input DTM (used for the simulation) and the raw simulated data.

- Fig. 5.1 shows a classical blue-colored style of water heights.

- At this scale, flow directions and velocities are represented by the color wheel presented above (Fig. 5.2.a).

- At a higher level of zoom, the graphic style is automatically modified to have a more suitable graphic representation of flow directions and velocities based on arrows, in order to help to pinpoint detected inconsistencies (Fig. 5.2.b)...

- ...Until a more zoomed graphic representation optimizes the number of displayed arrows and their color (Fig. 5.2.c).
Fig. 5.2.c again illustrates an inconsistency in flow simulation data, here due to too concentrated injection of discharge in the simulation domain.

\subsection{Automation in QGIS for use by hydraulics researchers}

Hydraulics researchers need to assign a specified style to a layer they need to analyze. They have to benefit from a predefined set of graphic styles, expected to facilitate their analysis process. The efficiency in the visual analysis of simulation results relies both in the combination of simulation outputs (i.e. interpret unusual heights by looking at directions in the same area) and in the co-visualization of those outputs together with geographic data describing the spatial configuration of the territory.

We resorted to QGIS styles and models (QGIS Development Team, 2009) for almost all co-designed graphic styles, previously considered useful by hydraulics researchers: our multiscale style presented in Fig.5, requires effectively many operations to be performed before reaching the desired display. This automation into QGIS will ensure that hydraulics specialists can use easily the set of graphic styles.

QGIS styles are pre-defined ways of displaying a data set on the user screen. For raster data sets, hue, intensity and/or transparency parameters can be assigned to cell values. For vector data sets, styles can also determined shapes, sizes and/or orientation of each objects.

QGIS models allow to define an entire workflow that includes pre-processing operations for both simulation results and other geographic data (filter, conditional selection and/or computation to combine several datasets, etc.), storage of needed intermediate results, and finally application of desired styles to each of the data sets. This includes the desired scale-range, since multi-scale styles require several styles for the same layer depending on the level of zoom.

Finally, we provided hydraulic researchers with a set of folders and files containing styles and models, a documentation of each of those styles and models and their use and a basic tutorial on how to use them. They have been effectively tested by hydraulic researchers and can be now used on new versions of simulation data.

\section{CONCLUSION AND PERSPECTIVES}

In this paper, we present our contribution to help researchers explore their hydraulic simulation results, based on a set of graphic multi-scale 2D styles. Our purpose in this interdisciplinary and iterative approach is to enrich knowledge and methods for visual analysis of simulated phenomena on earth. The interaction between geovisualization and hydraulics researchers indeed helped to set up graphic multi-scales styles, specifically designed for the diagnostic of hydraulic simulation results, and particularly the detection of inconsistencies. Although these styles were developed for the very specific simulation framework developed in the PICS project, they can be adapted for a broader use in the field of analysis of hydraulic simulation results, since many simulation models produce data similar to those from the PICS project, namely water heights, flow velocities and flow directions.

As far as the use of 2D geovisualizations to support visual analysis of simulation results is concerned, we have identified several issues that need to be addressed, in particular the development of automated and efficient geovisualizations allowing 

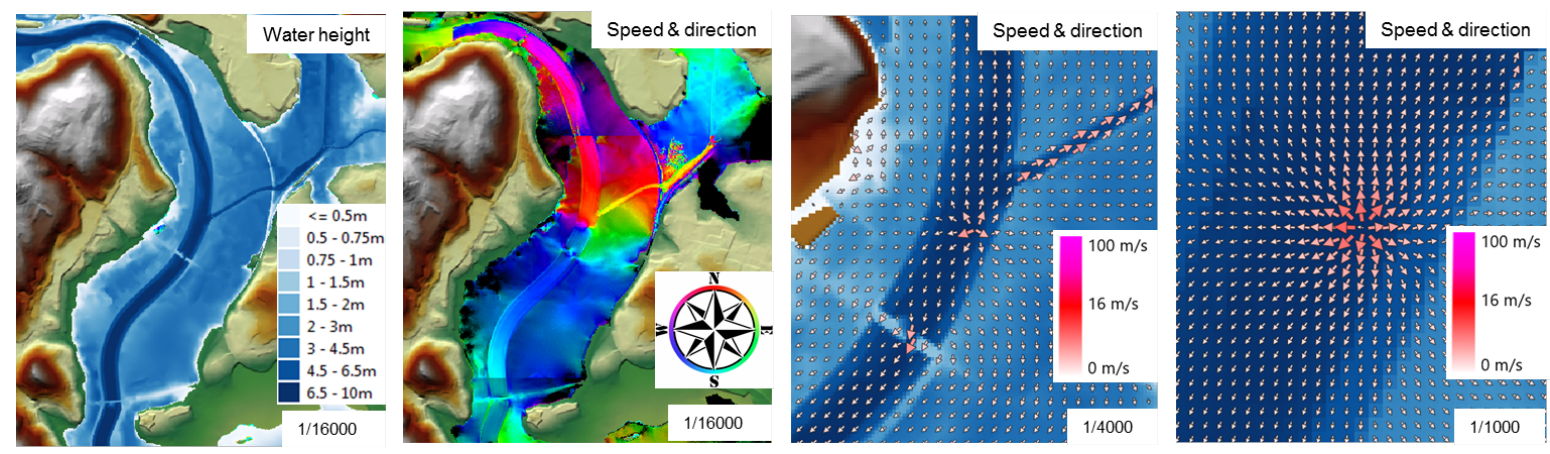

Figure 5. Multiscale styles for hydraulic data visualization: 1- Water heights with a proper colormap (classical blue style), 2- Merged flow directions and velocities: with a color wheel (a), with a first level of zoom based on arrows (b), with a second level of zoom based on arrows (c) (automated multi-scale style).

for the comparison of two, or more, simulation results on the same area produced with different parameters or input data by shedding light on their differences and the representation of uncertainties through the combined visualization of outputs from several scenarios over the same area.

We attempt now to pursue this interdisciplinary research work by designing and implementing the co-visualization of two flood simulation scenarii, in order to support comparison, similarities and differences detection, uncertainties revealed by the exploration of various hypothesis (minimum, average, maximum) for a scenario.

Thanks to the feedbacks from hydraulics researchers, we also established that basic 3D visualization of DTMs was indeed useful to confirm or infirm a visual interpretation of the topography and to help distinguishing between a spike or a hole in the terrain, or to better visualize the shape of the river bed cross sections in areas where the DTM appears to be very largely interpolated. At the end of the project, we briefly experimented co-visualization of $2 \mathrm{D}$ flow directions and velocities with arrows into a 3D urban model, in order to help detect impacted buildings for instance (Fig. 6). In-depth experimentations with those proposed visuals are planned next, and require many iterations between hydraulics and geovisualization researchers. Therefore, beyond their use for better relief understanding, 3D geovisualizations still need further testing to determine guidelines to ensure their usefulness for the analysis of simulation results.

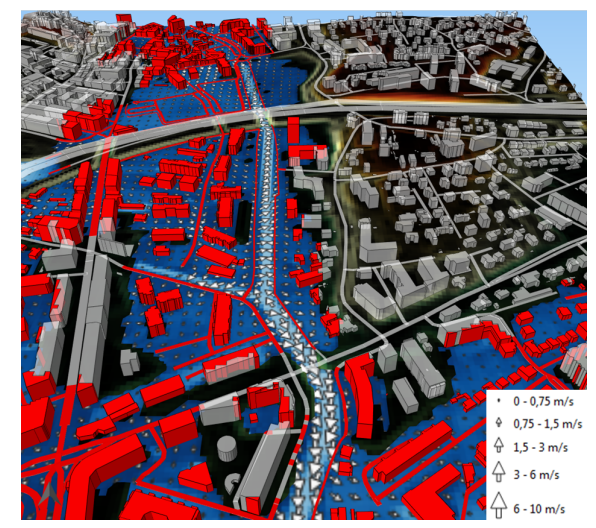

Figure 6. Simulation data interacting with 3D city model. In red, parts of buildings being impacted by simulated water flows.

\section{ACKNOWLEDGEMENTS}

This research work has been granted by the Urba Risk Lab (URL) project ${ }^{4}$. The hydraulic simulation data has been provided by the PICS research project, funded by the french national research agency (ANR), grant number ANR-17-CE030011.

\section{REFERENCES}

Bishop, I. D., Lange, E., 2005. Visualization in landscape and environmental planning : technology and applications. Taylor \& Francis, London.

Brasebin, M., Christophe, S., Jacquinod, F., Vinesse, A., Mahon, H., 2016. 3D Geovisualization and Stylization to Manage Comprehensive and Participative Local Urban Plans. ISPRS Annals of Photogrammetry, Remote Sensing and Spatial Information Sciences, IV-2/W1, 83-91.

Carrillo, E., Verna, R., Noriega, G., Benthien, M. L., 2019. A story of california's seismic hazards: Studying historical and simulated earthquakes through geovisualization platforms. AGU Fall Meeting Abstracts.

Davy, P., Croissant, T., Lague, D., 2017. A precipiton method to calculate river hydrodynamics, with applications to flood prediction, landscape evolution models, and braiding instabilities. Journal of Geophysical Research-Earth Surface, 122(8), 14911512.

Goda, K., Song, J., 2016. Uncertainty modeling and visualization for tsunami hazard and risk mapping: a case study for the 2011 Tohoku earthquake. Stochastic Environmental Research and Risk Assessment, 30(8), 2271-2285.

Jacquinod, F., 2014. Production, pratique et usages des géovisualisations $3 \mathrm{~d}$ dans l'aménagement du territoire, $\mathrm{PhD}$ thesis, Saint-Etienne University.

Jacquinod, F., Bonaccorsi, J., 2019. Studying Social Uses of 3D Geovisualizations: Lessons Learned from Action-Research Projects in the Field of Flood Mitigation Planning. ISPRS International Journal of Geo-Information, 8(2), 84.

Jacquinod, F., Pedrinis, F., Edert, J., Gesquière, G., 2016. Automated production of interactive $3 \mathrm{~d}$ temporal geovisualizations

\footnotetext{
$\overline{{ }^{4} \text { https://urbarisklab.org/en/ }}$
} 
so as to enhance flood risk awareness. V. Tourre, F. Biljecki (eds), Eurographics Workshop on Urban Data Modelling and Visualisation, The Eurographics Association.

Kemec, S., Duzgun, S., Zlatanova, S., Dilmen, D. I., Yalciner, A. C., 2010. Selecting 3d Urban Visualisation Models For Disaster Management: Fethiye Tsunami Inundation Case. 9.

Kilsedar, C. E., Fissore, F., Pirotti, F., Brovelli, M. A., 2019. Extraction and Visualization of 3D Building Models in Urban Areas for Flood Simulation. ISPRS - International Archives of the Photogrammetry, Remote Sensing and Spatial Information Sciences, XLII-2/W11, 669-673.

Klockow-McClain, K. E., McPherson, R. A., Thomas, R. P., 2020. Cartographic Design for Improved Decision Making: Trade-Offs in Uncertainty Visualization for Tornado Threats. Annals of the American Association of Geographers, 110(1), 314-333.

Kostelnick, J. C., McDermott, D., Rowley, R. J., Bunnyfield, N., 2013. A Cartographic Framework for Visualizing Risk. Cartographica: The International Journal for Geographic Information and Geovisualization, 48(3), 200-224.

Kübler, I., Richter, K.-F., Fabrikant, S. I., 2019. Against All Odds: Multicriteria Decision Making with Hazard Prediction Maps Depicting Uncertainty. Annals of the American Assoc. of Geographers, 0(0), 1-23.

Leskens, J. G., Brugnach, M., Hoekstra, A. Y., 2014. Application of an Interactive Water Simulation Model in urban water management: a case study in Amsterdam. Water Science \& Technology, 70(11), 1729.

Leskens, J. G., Kehl, C., Tutenel, T., Kol, T., Haan, G. d., Stelling, G., Eisemann, E., 2017. An interactive simulation and visualization tool for flood analysis usable for practitioners. Mitigation and Adaptation Strategies for Global Change, 22(2), 307-324.

Lieske, D. J., 2012. Towards a Framework for Designing Spatial and Non-Spatial Visualizations for Communicating Climate Change Risks. Geomatica, 66(1), 10.

Lim, N. J., 2018. Modelling, mapping and visualisation of flood inundation uncertainties, $\mathrm{PhD}$ thesis, Gävle University.

Lonergan, C., Hedley, N., 2015. Navigating the future of tsunami risk communication: using dimensionality, interactivity and situatedness to interface with society. Natural Hazards, 78(1), 179-201.

Massaâbi, M., Layouni, O., Oueslati, W. B. M., Alahmari, F., 2018. An immersive system for $3 \mathrm{~d}$ floods visualization and analysis. D. Beck, C. Allison, L. Morgado, J. Pirker, A. Peña-Rios, T. Ogle, J. Richter, C. Gütl (eds), Immersive Learning Research Network, 840, Springer International Publishing.

Masse, A., Christophe, S., 2015. Homogeneous geovisualization of coastal areas from heterogeneous spatio-temporal data. International Archives of the Photogrammetry, Remote Sensing and Spatial Information Sciences, XL-3/W3, 509-516.

Padilla, L., Creem-Regehr, S., Hegarty, M., Stefanucci, J., 2018. Decision making with visualizations: a cognitive framework across disciplines. Cognitive Research: Principles and Implications, 3.
Patel, V. M., Dholakia, M. B., Singh, A. P., 2013. Tsunami Risk 3D Visualizations of Okha Coast, Gujarat (India). International Journal of Engineering Science and Innovative Technology, 2(2), 9.

Pierkot, C., Christophe, S., Girres, J.-F., 2019. Exploring multiplexing tools for co-visualization in crisis units. 16th International Conference on Information Systems for Crisis Response and Management (ISCRAM 2019), Valencia, Spain.

QGIS Development Team, 2009. QGIS Geographic Information System Manual. Open Source Geospatial Foundation.

Saha, S., Shekhar, S., Sadhukhan, S., Das, P., 2018. An analytics dashboard visualization for flood decision support system. Journal of Visualization, 21(2), 295-307.

Seipel, S., Lim, N. J., 2017. Color map design for visualization in flood risk assessment. International Journal of Geographical Information Science, 31(11), 2286-2309.

Sheppard, S. R. J., 2005. Validity, reliability and ethics in visualization. I. Bishop, E. Lange (eds), Visualization in Landscape and Environmental Planning - Theory and Applications, Taylor and Francis, 79-97.

Yang, B., 2016. GIS based 3-D landscape visualization for promoting citizen's awareness of coastal hazard scenarios in flood prone tourism towns. Applied Geography, 76, 85-97. 